\title{
PIEZOELECTRIC AND PYROELECTRIC PROPERTIES OF PT/P(VDF-TrFE) 0-3 COMPOSITES
}

H.L.W. CHAN, W.K.CHAN, C.L. CHOY and Deming LEI

Department of Applied Physics and Materials Research Centre,

The Hong Kong Polytechnic University, Hung Hom, Kowloon, Hong Kong

Abstract

Lead titanate (PT)/vinylidene fluoride-trifluoroethylene (P(VDF-TrFE)) copolymer 0-3 composites have been fabricated by solution blending followed by compression-molding. In order to obtain piezoelectric and pyroelectric activities the composites have been polarized by a stepwise poling method. Samples with (i) polarization in the ceramic phase only and (ii) parallel polarization in the ceramic and copolymer have been prepared. The piezoelectric and pyroelectric coefficients of these two types of composites have been measured and the results are discussed.

\section{Introduction}

Conventional ferroelectric ceramics such as lead zirconate titanate (PZT) and modified lead titanate (PT) are popular choices in piezoelectric or pyroelectric sensor applications. These materials have high thickness electromechanical coupling coefficients $\left(k_{t}\right)$, a wide range of dielectric permittivity and low dielectric and mechanical losses. However, their high acoustic impedances result in poor acoustic coupling to water or human tissues. Ferroelectric polymers such as polyvinylidene fluoride (PVDF) and vinylidene fluoride/trifluoroethylene (P(VDFTrFE)) have a low acoustic impedance but also have low $\mathrm{k}_{\mathrm{t}}$ and $\varepsilon$ and high dielectric losses. $\mathrm{PT} / \mathrm{P}(\mathrm{VDF}-\mathrm{TrFE})$ ) $0-3$ composites have attracted considerable reseach interests $[1,3]$ as they offer properties intermediate between $\mathrm{PT}$ and $\mathrm{P}(\mathrm{VDF}-\mathrm{TrFE})$. After combining the two phases in a 0-3 connectivity, the composite must be poled by exposure to a high electric field. In the present study, electric field was first applied at a temperature above the Curie temperature for heating $\left(\uparrow T_{c}\right)$ of the copolymer in order to polarize the ceramic. After cooling to room temperature the composites were heated to a temperaure below $\uparrow T_{c}$ and then poled again by an electric field aligned parallel to the polarization of the ceramic. The piezoelectric and pyroelectric properties of these composites were studied.

\section{Sample fabrication}

The P(VDF-TrFE) copolymer pellets supplied by Piezotech have a nominal TrFE content of $35 \mathrm{~mol} \%$. The endotherms of the as-supplied pellets was measured at a heating rate of $10^{\circ} \mathrm{C} / \mathrm{min}$ and at a cooling rate of $-10^{\circ} \mathrm{C} / \mathrm{min}$ using a Perkin Elmer DSC7 Thermal Analyzer. The Curie temperature of the copolymer for heating $\uparrow T_{c}$ was $102^{\circ} \mathrm{C}\left(\uparrow T_{c}=101.7^{\circ} \mathrm{C}\right.$ for first heating and $\uparrow T_{c}=102.5^{\circ} \mathrm{C}$ for second heating) and $\downarrow T_{c}=62^{\circ} \mathrm{C}$ upon cooling. The melting temperature $T_{m}$ was $153^{\circ} \mathrm{C}$

The calcium modified lead titanate $\left(\left(\mathrm{Pb}_{0.76} \mathrm{Ca}_{0.24}\right)\left[\left(\mathrm{Co}_{0.5} \mathrm{~W}_{0.5}\right)_{0.04} \mathrm{Ti}_{0.96}\right] \mathrm{O}_{3}\right)$ was supplied by Zhongshan University in China and was prepared by the conventional ball-milling mixed 
oxide process. It was calcined at $900^{\circ} \mathrm{C}$ for 2 hours and sintered at $1130^{\circ} \mathrm{C}$ for about 7 hours. The PT ceramic powder was obtained by grinding a sintered ceramic block in a ball-milling machine.

A predetermined amount of copolymer pellets was dissolved in acetone at room temperature and the required quantity of PT powder was blended into the solution. The mixture was then poured onto a petri-dish and after evaporation of the solvent in air, the mixture was placed in an oven at $120^{\circ} \mathrm{C}$ for 2 hours to ensure that the solvent has complctely evaporated. The dried composite was cut into small pieces and was molded into dise shape by pressing in a compression machine at $200^{\circ} \mathrm{C}$ and at a pressure of $48.8 \mathrm{~kg} / \mathrm{mm}^{2}$. Composite discs with various volume fractions of ceramic $\phi$ and of thickness $0.5 \mathrm{~mm}$ and diameter $12.8 \mathrm{~mm}$ were prepared using the method described above.

\section{Relative permittivity measurements}

The relative permittivities of the unpoled 0-3 composite samples were measured as functions of temperature at $1 \mathrm{kHz}$ using an HP $4194 \mathrm{~A}$ impedance analyzer. The sample was heated or cooled at a rate of about $1^{\circ} \mathrm{C} / \mathrm{min}$ in a temperature controlled oven until a certain temperature was reached, and then kept at that temperature for half an hour before measurement was made. The resulting relative pernaittivities for PT, P(VDF-TrFE) and composites with various volume fractions of ceramic $\phi$ are shown as functions of temperature in Fig.1. The relative permittivity of PT (Fig. 1a) is about 250 at $30^{\circ} \mathrm{C}$ and increases roughly linearly with temperature at a rate of $0.43 \%$ per degree. Thermal hysteresis was observed for the copolymer and 0-3 composites and abrupt changes in the relative permittivities occurred at different temperatures upon heating and cooling.
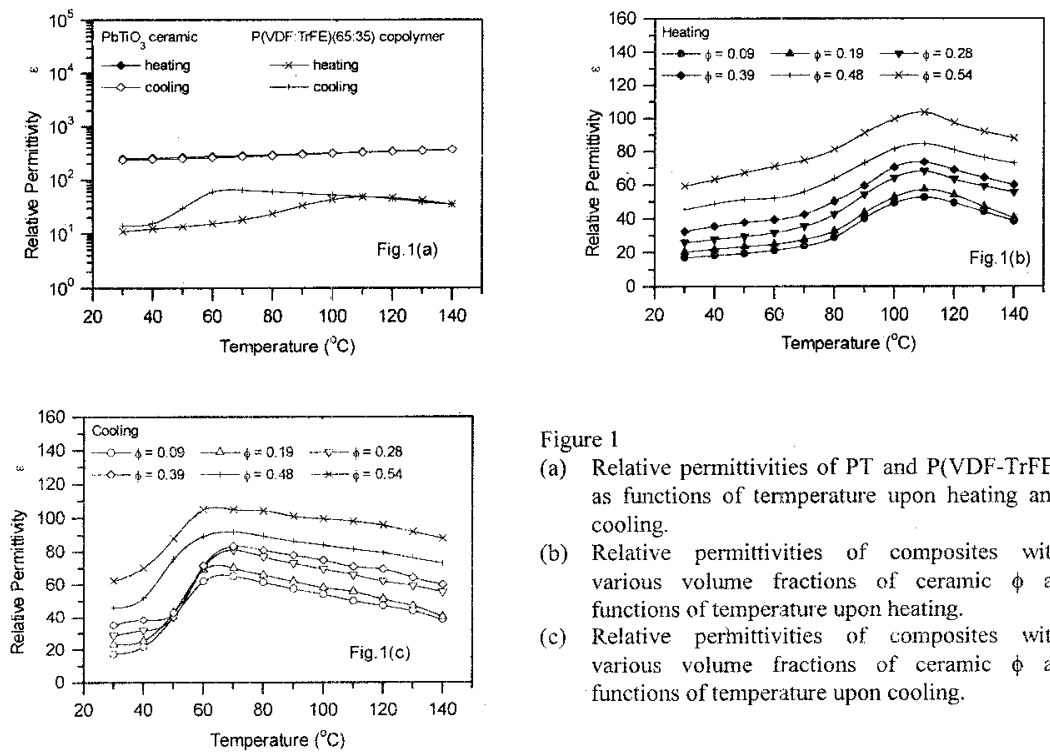

Figure 1

(a) Relative permittivities of $\mathrm{PT}$ and $\mathrm{P}(\mathrm{VDF}-\mathrm{TrFE})$ as functions of temperature upon heating and cooling.

(b) Relative permittivities of composites with various volume fractions of ceramic $\phi$ as functions of temperature upon heating.

(c) Relative permittivities of composites with various volume fractions of ceramic $\phi$ as functions of temperature upon cooling. 
Similar to the results reported for lead zirconate titanate/vinylidene fluoride-trifluoroethylene $0-3$ composites [4], below the Curie temperature of the copolymer, the relative permittivities of $\mathrm{PT} / \mathrm{P}$ (VDF-TrFE) 0-3 composites (shown in Fig. 2) agree reasonably well with the values calculated using the Bruggeman equation $[5,6]$ :

$$
\left(\varepsilon_{c}-\varepsilon\right) /(\varepsilon)^{1 / 3}=(1-\phi)\left(\varepsilon_{c}-\varepsilon_{p}\right) /\left(\varepsilon_{p}\right)^{1 / 3}
$$

where $\varepsilon_{c}, \varepsilon_{p}$ and $\varepsilon$ are the dielectric constants of the ceramic, copolymer and composite, respectively.
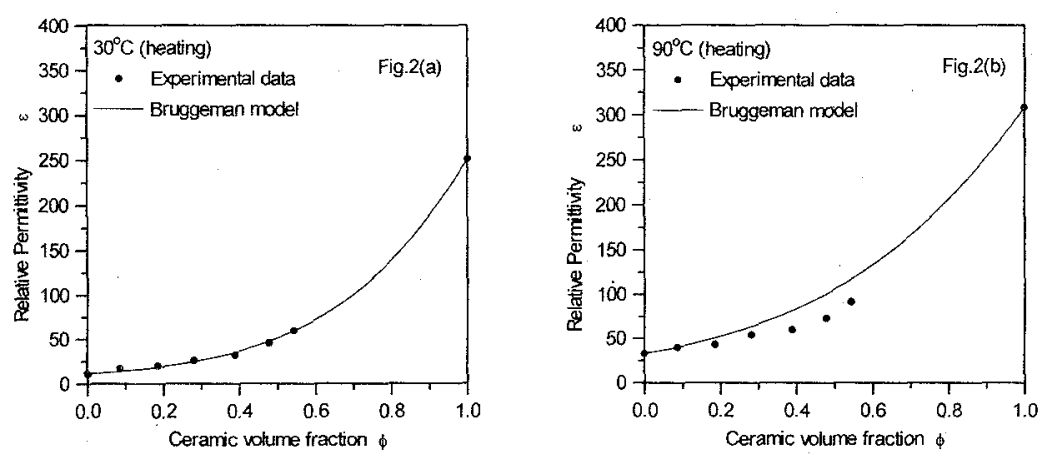

Figure 2 (a) The relative permittivity of PT/P(VDF-TrFE) $0-3$ composites as a function of the volume fraction of ceramic $\phi$ at $30^{\circ} \mathrm{C}$ upon heating.

(b) The relative permittivity of PT/P(VDF-TFE) 0-3 composites as a function of the volume fraction of ceramic $\phi$ at $90^{\circ} \mathrm{C}$. upon heating.

\section{Electric field acting on the PT particles inside a composite}

After a d.c. electric field has been applied to a composite for a time long compared to the interfacial relaxation time, the ratio of the electric field in the two phases estimated from the Maxwell-Wagner model is given by $E_{d} / E_{p}=\rho_{c} / \rho_{p},[2,3]$ where $\rho_{c}$ and $\rho_{p}$ are the resistivity of the ceramic and the copolymer, respectively. For a time short compared to the interfacial relaxation time, the electric field ratio is dertermined by the relative permittivities: $E_{c} / E_{p}=$ $\varepsilon_{\mathrm{p}} / \varepsilon_{\mathrm{c}} \cdot[2,3]$, For a composite containing a spherical particle of dielectric constant $\varepsilon_{\mathrm{c}}$ embedded in a continuous medium of relative permittivity $\varepsilon$, the local field experienced by the particle is given by [7]

$$
\mathrm{E}_{\mathrm{c}}=3 \varepsilon \mathrm{E}_{\mathrm{o}} /\left(2 \varepsilon+\varepsilon_{\mathrm{c}}\right)
$$

where $E_{o}$ is the applied electric field. The observed values of $\varepsilon$ were used in eqn. (2) to calculate $\mathrm{E}_{c} / \mathrm{E}_{0}$. As shown in Fig. 3 , at temperatures above $90^{\circ} \mathrm{C}$, the electric field acting on the PT particles in a composite with a ceramic volume fraction of 0.48 is as high as 0.5 of the applied field. 


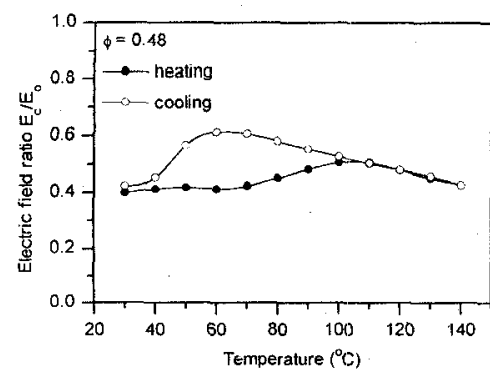

5. Poling of PT/P(VDF-TrFE) 0-3 composites

\subsection{Poling of the PT ceramic}

Attempts were made to polarize the composites in the regime where the electric field ratio was determined by the relative permittivities. Composites with $\phi=0.48$ were heated to $120^{\circ} \mathrm{C}$ (which is above $\uparrow T_{c}$ of the copolymer) and stepwise poling method [8] was used. The electric field was increased from 0 to $E_{1}$ and kept at $E_{1}$ for a time $t_{p}(\sim 4 . \min$.); the field was then switched off and the sample short-circuited for time $t_{s}(\sim 8 \mathrm{~min}$.). Electric field was then increased to $E_{2}\left(>E_{1}\right)$ and kept constant for time $t_{p}$ and then the field was switched off and the sample short-circuited for a time $t_{s}$. The electric field was increased after each short-circuited step and a field as high as $27 \mathrm{kV} / \mathrm{mm}$ can be reached. With this stepwise process, the risk of electric breakdown was reduced. This may result from the short-circuted periods during which the mechanical stress and the injected space charges were partially relaxed, thereby leading to a more homogeneous electric field distribution.

Since the electric field was switched off at $120^{\circ} \mathrm{C}$ (above the Curie temperature of the copolymer) and the composites were cooled to room temperature under zero field, only the ceramic phase was polarized and contribued to the pyroelectric and piezoelectric properties.

After cooling to room temperature, the ceramic poling ratio $\alpha$ was estimated from the peak heights $\mathrm{I}_{(200)}$ and $\mathrm{I}_{(002)}$ of the (200) and (002) x-ray reflections arising from the PT ceramic[9]

$$
\alpha=\mathrm{I}_{(002)} /\left[\mathrm{I}_{(002)}+\mathrm{I}_{(200)}\right]
$$

\subsection{Poling of the copolymer}

Composites with a polarized PT ceramic phase were heated to $75^{\circ} \mathrm{C}$ and poled again by the stepwise poling method and with the polarity of the field applied to the sample in a direction parallel to the polarization of the ceramic. A maximum field of $27 \mathrm{kV} / \mathrm{mm}$ could be applied. The samples were short-circuited and annealed at $70^{\circ} \mathrm{C}$ for $15 \mathrm{~h}$ before measurement. 


\section{Measurement of piezo- and pyro-electric coefficients}

The impedance of the samples was measured using an HP 4194A impedance analyzer and the thickness mode electromechanical coupling coefficient $k_{t}$ calculated from the impedance according to the IEEE standard [10]. The pyroelectric coefficient $p$ was measured using the digital integration method. [11] The $d_{33}$ coefficient of the samples was determined using a Pennebaker model 8000 piezo $\mathrm{d}_{33}$ tester from American Piezo-Ceramics, Inc.. The measured properties of the composite samples together with the properties of PT and the copolymer were tabulated in Table 1 .

As shown in Table 1, the copolymer has a $\mathrm{d}_{33}$ value smaller than PT but of opposite sign, hence when both phases are polarized, the piezoelectric properties cancel each other resulting in lower $d_{33}$ and $k_{t}$ values. Unlike the piezoelectric coefficient, the pyroelectric coefficient increased by $50 \%$ after the copolymer have been poled in the same direction as PT.

Table 1 Piezo and pyro-electric properties of PT, P(VDF-TrFE) and PT/P(VDF-TrFE) 0-3 composite with $\phi=0.48$.

\begin{tabular}{|l|c|c|c|c|}
\hline \multicolumn{1}{|c|}{ Sample } & poling ratio $\alpha$ & $\mathrm{d}_{33}(\mathrm{pC} / \mathrm{N})$ & $\mathrm{k}_{\mathrm{t}}$ & $\mathrm{p} \mu \mathrm{C} / \mathrm{m}^{2} \mathrm{~K}\left(25^{\circ} \mathrm{C}\right)$ \\
\hline Lead titanate PT & 0.75 & 61.9 & 0.40 & 152.1 \\
\hline 65/35 P(VDF-TrFE) & ---- & -37.2 & 0.21 & 25.7 \\
\hline $\begin{array}{l}\text { Composite: with polarized } \\
\text { PT }\end{array}$ & 0.47 & 20.4 & 0.17 & 40.7 \\
\hline $\begin{array}{l}\text { Composite: field applied to } \\
\text { P(VDF-TrFE) parallel to } \\
\text { field applied to PT }\end{array}$ & 0.42 & 15.4 & 0.15 & 60.3 \\
\hline
\end{tabular}

\section{Laser-induced pressure pulse measurements}

The laser induced pressure pulse (LIPP) method [12] was used to determine the polarization distribution in the samples and to distinguish whether the piezoelectric property arose from the ceramic or the copolymer. As the piezoelectric coefficient $e_{33}$ of PT and the copolymer have opposite signs, the pressure pulses generated would have opposite polarity, with the signal for PT larger than that of the copolymer. After the composite had been poled at $120^{\circ} \mathrm{C}$, a large negative pressure pulse was detected at the anode side (the side connected to positive poling voltage) and the LIPP signal indicated that the sample was uniformly poled, with the piezoelectric coefficient $\left(\mathrm{e}_{33}\right)_{1}$ contributed mainly by PT. After the composite was poled again at $75^{\circ} \mathrm{C}$ with the applied field parallel to the polarization of the PT phase, the LIPP signal became smaller, implying a reduction in the piezoelectric coefficient due to the negative contribution from the polarized copolymer matrix. To verify this further, the sample with both phases polarized was annealed at $120^{\circ} \mathrm{C}$ for $15 \mathrm{~h}$ to remove the polarization of the copolymer and the LIPP measurement was repeated. The annealed sample was found to have a piezoelectric coefficient almost the same as the coefficient prior to poling of the copolymer. 


\section{Conclusion}

The permittivity $\varepsilon$ of PT/P(VDF-TrFE) 0-3 composites have been determined from $20^{\circ} \mathrm{C}$ to $140^{\circ} \mathrm{C}$. The observed $\varepsilon$ agree quite well with the predictions of the Bruggeman model. The polarization distribution in a PT/P(VDF-TrFE) 0-3 composite with a ceramic volume fraction of 0.48 was determined by the laser-induced pressure pulse method. It was found that a polarized copolymer matrix gave rise to a reduction in the piezoelectric coefficients but an increase in the pyroelectric coefficient. Studies on the piezoelectric and pyroelectric properties of PT/P(VDF-TrFE) composites with other volume fractions of ceramic are in progress.

\section{ACKNOWLEDGEMENTS}

The authors would like to acknowledge the financial support from the Hong Kong Research Grant Council. Thanks are also due to Dr. Y. Zhang for his assistance in the LIPP measurements.

\section{REFERENCES}

[1] R.E. Newnham, D.P. Skinner, K.A. Klicker, A.S. Bhalla, B. Hardiman and T.R. Gururaja, "Ferroelectric ceramic-plastic composites for piezoelectric and pyroelectric applications", Ferroelectrics, Vol. 27, pp. 49-55, 1980.

[2] M.H. Lee, A. Halliyal and R.E. Newnham, "Poling of coprecipitated lead-titanate-epoxy 0-3 piezoelectric composites", J. Am. Ceram. Soc., Vol. 72, 986-990, 1989.

[3] C. Dias and D.K. Das-Gupta, Key Engineering Materials Vols. 92-93, Ferroelectric Polymers and Ceramic-Polymer Composites, Trans Tech Publications, Aedermannsdorf, Switzerland, 1994, Chapter 8.

[4] H.L.W. Chan, Y. Chen and C.L. Choy, "Thermal Hysteresis in the Relative permittivities and the Polarization of Lead Zirconate/Vinylidene FluorideTrifluoroethylene 0-3 Composites", To be published in IEEE. Trans. Diel. Electr. Insul..

[5] L.K.H. van Beek, "Dielectric behaviour of heterogeneous systems", Progress in Dielectrics, vol. 7 , Heywood, London, 1967, pp,69-114.

[6] D.A.G. Bruggeman, Ann. Phys. Lpz. Vol. 24, 636, 1935.

[7] L.D. Landau and E.M. Lifshitz, Course of Theoretical Physics, Vol. 8, Electrodynamics of continuous media, Pergamon Press, U.K.,1960, Chapter 11.

[8] D. Setiadi, M. Wubbenhorst and P.P.L. Regtien, "The stepwise poling of a thin VDF/TrFE copolymer", Proc. 8th European meeting on ferroelectricity, Nijmegen, The Netherlands, July, 4-8, 1995, pp. 14-18.

[9] K. F. Schoch Jr., D.P. Partlow and R.E. Krause, "Assessment of degree of poling in 0-3 piezoelectric composites by x-ray methods", Ferroelectrics, Vol. 77, 39-46, 1988.

[10] IEEE Standard on Piezoelectricity, IEEE standard 176, 1978.

[11] Y. Xu,"Ferroelectric Materials and Their Applications", (Elsevier, New York, 1991).

[12] C. Alquie, J. Lewiner, "A New Method for Studying Piczoelectric Materials", Rev. Phys. Appl., Vol. 20, pp. 395-402, 1985. 